\title{
Targeting NLRP3 Inflammasome in the Treatment of CNS Diseases
}

\author{
Bo-Zong Shao, Qi Cao and Chong Liu* \\ Department of Pharmacology, Second Military Medical University, Shanghai, China
}

Central nervous system (CNS) is one of the largest killers of people's health all over the world. The overactivation of the immune and inflammatory responses is considered as an important factor, contributing to the pathogenesis and progression of CNS disorders. Among all kinds of immune and inflammatory reaction, the inflammasome, a complex of proteins, has been drawn increasingly attention to by researchers. The initiation and activation of the inflammasome is involved in the onset of various kinds of diseases. The NLRP3 inflammasome, the most studied member of the inflammasome, is closely associated with many kinds of CNS disorders. Here in this review, the roles of the NLRP3 inflammasome in the pathogenesis and progression of several well-known CNS diseases would be discussed, including cerebrovascular diseases, neurodegenerative diseases, multiple sclerosis, depression as well as other CNS disorders. In addition, several therapeutic strategies targeting on the NLRP3 inflammasome for the treatment of CNS disorders would be described in this review.

Keywords: NLRP3 inflammasome, CNS diseases, innate immunity, inhibitors, pharmacological application

\section{OPEN ACCESS INTRODUCTION}

Edited by:

Juan Pablo De Rivero Vaccari,

University of Miami, United States

Reviewed by:

Denis Gris,

Université de Sherbrooke, Canada Sophie Anne Georgin-Lavialle,

Université Pierre et Marie Curie,

France

*Correspondence:

Chong Liu

wanlc2004@aliyun.com

Received: 09 May 2018

Accepted: 17 August 2018

Published: 04 September 2018

Citation:

Shao B-Z, Cao Q and Liu C (2018) Targeting NLRP3 Inflammasome in the Treatment of CNS Diseases.

Front. Mol. Neurosci. 11:320. doi: 10.3389/fnmol.2018.00320
Innate immunity is a vital self-defensive mechanism against various kinds of internal and external threatening factors in organism (Thaiss et al., 2016; Leentjens et al., 2018; Suslov et al., 2018; Williams and O'Neill, 2018). The inflammasome is involved in the innate immune reaction, which, according to the name, is also defined as a special type of protein complex associated with inflammatory reaction, thus serving as connection between immune and inflammatory responses (Awad et al., 2018; Shibata, 2018). Among all kinds of inflammasomes, the NOD-like receptor family, pyrin domain containing 3 (NLRP3) inflammasome is the most studied one, which is involved in the pathogenesis and progression of various kinds of immune- and inflammationrelated disorders, including central nervous system (CNS) diseases (Du et al., 2018; Gong et al., 2018; He et al., 2018; Sharma et al., 2018). So far, various kinds of therapeutic pathways taking advantage of the inhibition of the NLRP3 inflammasome have been developed or studied in the treatment of diseases based on our current knowledge on the NLRP3 inflammasome in diseases (Birnbaum et al., 2018; Mangan and Latz, 2018; McAllister et al., 2018; Singh and Jha, 2018). CNS diseases refer to a group of pathological processes occurring in CNS, leading to the damage of neural function or structure (Cacabelos et al., 2016). The overreaction of inflammatory and immune reaction has been considered as an important factor in the initiation and progression of various kinds of CNS diseases. Thus, targeting on the inhibition of inflammatory and immune reaction in CNS could contribute greatly to the alleviation of CNS diseases (Anuncibay-Soto et al., 2018; Rayasam et al., 2018; Sekerdag et al., 2018; Stephenson et al., 2018). Here in this review, we will discuss the roles of the NLRP3 inflammasome in several kinds of CNS disorders including 
cerebrovascular diseases, neurodegenerative diseases, multiple sclerosis, depression, and other CNS disorders such as traumatic brain injury. In addition, several therapeutic strategies against CNS diseases taking advantage of the NLRP3 inflammasome inhibition will be further discussed in this current review, aiming to illustrate a whole picture on the current knowledge and application of the NLRP3 inflammasome inhibitors in the treatment of CNS diseases.

\section{NLRP3 INFLAMMASOME}

The inflammasome, an inducer of innate immune reaction, functions in the recognition and targeting of numerous invasive or internal pathogens such as microbes (Malik and Kanneganti, 2017; Lugrin and Martinon, 2018). It is widely acknowledged that the inflammasomes are mainly produced in immune and inflammatory cells including macrophages, $\mathrm{T}$ lymphocytes and $\mathrm{N} \& \mathrm{~K}$ cells, contributing to the triggering of the anti-pathogen immune inflammatory responses (Speeckaert et al., 2016; Correada-Silva et al., 2017; Di Virgilio et al., 2017). So far, several forms of inflammasomes have been described, mainly including the NLRP1, NLRP2, NLRP3, double-stranded DNA sensors absent in melanoma 2 (AIM2) as well as NLRC4 inflammasome (Ozaki et al., 2015; Duncan and Canna, 2018). Among them, the NLRP3 inflammasome is the most characterized and studied one, which has been demonstrated to be involved in the pathogenesis and progression of various kinds of diseases (Heo et al., 2018; Sano et al., 2018; Xiao et al., 2018; Zhai et al., 2018). Hereafter, the components and activation of the NLRP3 inflammasome will be discussed in the following contents.

The NLRP3 inflammasome is comprised of three components, including the NLRP3 protein, adapter protein apoptosisassociated speck-like protein (ASC), and procaspase-1 (Ito et al., 2015; Birnbaum et al., 2018). In the absence of activating factors such as pathogen-associated molecular patterns (PAMPs) and danger-associated molecular patterns (DAMPs), the leucine-rich repeats (LRRs) and NACHT domain in the NLRP3 protein connect with each other tightly to get rid of the interaction of NLRP3 protein and ASC (Shao et al., 2015; Kosmidou et al., 2018). Under the challenge of immune stimuli, the NLRP3 protein is activated, followed by the interaction with ASC and procaspase- 1 on the pyrin domain (PYD) and caspase recruitment domain (CARD) in the NLRP3 protein, respectively, thus leading to the assembly of the NLRP3 inflammasome (Shao et al., 2015; Gambin et al., 2018; Figure 1).

Generally speaking, the activation of the NLRP3 inflammasome involves two steps (Sutterwala et al., 2014; Shao et al., 2015; Wu et al., 2018a; Yuan et al., 2018; Figure 1). In the first step, a priming signaling is triggered by certain PAMPs or DAMPs on Toll-like receptor 4 (TLR4), which leads to the activation of the NF-кB-mediated pathway. The triggering of $\mathrm{NF}-\kappa \mathrm{B}$ activation leads to the increase in the transcription of the NLRP3 inflammasome-related components, including the NLRP3 protein, pro-interleukin-1 $\beta$ (proIL-1 $\beta$ ), and proIL-18. In the subsequent step two, with the further stimuli on immune and inflammatory cells, the NLPR3 protein was oligomerized, followed by the assembly of the NLRP3 protein, ASC and procaspase-1 into the complex of the NLRP3 inflammasome. The successful formation of the NLRP3 inflammasome triggers the transformation of the procaspase- 1 to caspase-1, catalyzing the formation of the mature IL- $1 \beta$ and IL- 18 from proIL- $1 \beta$ and proIL-18, which are secreted outside and lead to the cascade immune or inflammatory reaction (Place and Kanneganti, 2018; Shen et al., 2018).

So far, several factors have been demonstrated to lead to the activation of the NLRP3 inflammasome. For the initiation of the NLRP3 inflammasome, lipopolysaccharide (LPS) is widely considered to be a classic ligand for the activation of the TLR4 (Chu et al., 2018; Ho and Chang, 2018). In addition, several factors have been shown to be effective to induce the second step of the NLRP3 inflammasome activation, including the adenosine triphosphate (ATP, triggering the intracellular $\mathrm{K}^{+}$efflux), PAMPs, DAMPs, silica, $\beta$-amyloid, autophagy deficiency as well as factors leading to the enhancement of mitochondrial $\mathrm{Ca}^{2+}$ overload and so on (Shao et al., 2017b; Chen et al., 2018; Li et al., 2018b; Meng et al., 2018; Zhao et al., 2018).

\section{NLRP3 INFLAMMASOME IN CNS DISORDERS}

As we discussed above, the NLRP3 inflammasome is mainly produced in innate immunity cells, thus triggering the cascade immune and inflammatory reaction through the secretion of two pro-inflammatory cytokines including IL-1 $\beta$ and IL-18. As a result, the NLRP3 inflammasome is highly involved in the onset and development of various kinds of diseases, including cardiovascular diseases (myocardial ischemia/infarction, atherosclerosis, and hypertension), metabolic disorders (obesity, diabetes, and metabolic syndrome), digestive diseases (inflammatory bowel disease), renal diseases and CNS diseases (Chen et al., 2017; Kammoun et al., 2018; Martinez et al., 2018; Nasoohi et al., 2018; Sharma et al., 2018; Wu et al., 2018b; Yuan et al., 2018). In addition, it has been demonstrated that the polymorphism of the NLRP3 genes may lead to the occurrence of some congenital disorders in patients and animal models (Lewis et al., 2011; Kuemmerle-Deschner et al., 2017; Landmann and Walker, 2017). For example, it was reported that the cryopyrin-associated periodic fever syndrome (CAPS), a group of rarely diagnosed hereditary autoimmune diseases including Muckle-Wells syndrome and familial cold auto-inflammatory diseases, was mainly led to by the gain-of-function mutations of NLRP3 genes, which resulted in the overwhelming production and secretion of IL-1 $\beta$ and IL-18 (Kuemmerle-Deschner et al., 2017; Landmann and Walker, 2017). In CNS, it has been widely demonstrated that the NLRP3 inflammasome is highly involved in the pathogenesis and progression of various kinds of disorders, and strategies targeting on the inhibition of the NLRP3 inflammasome activation are increasingly developed and regarded as potential and effective pathways in the treatment of CNS diseases (de Rivero Vaccari et al., 2014; Zhou et al., 2016; Song et al., 2017). In this section, we will discuss the roles of the NLRP3 inflammasome in several well-known 


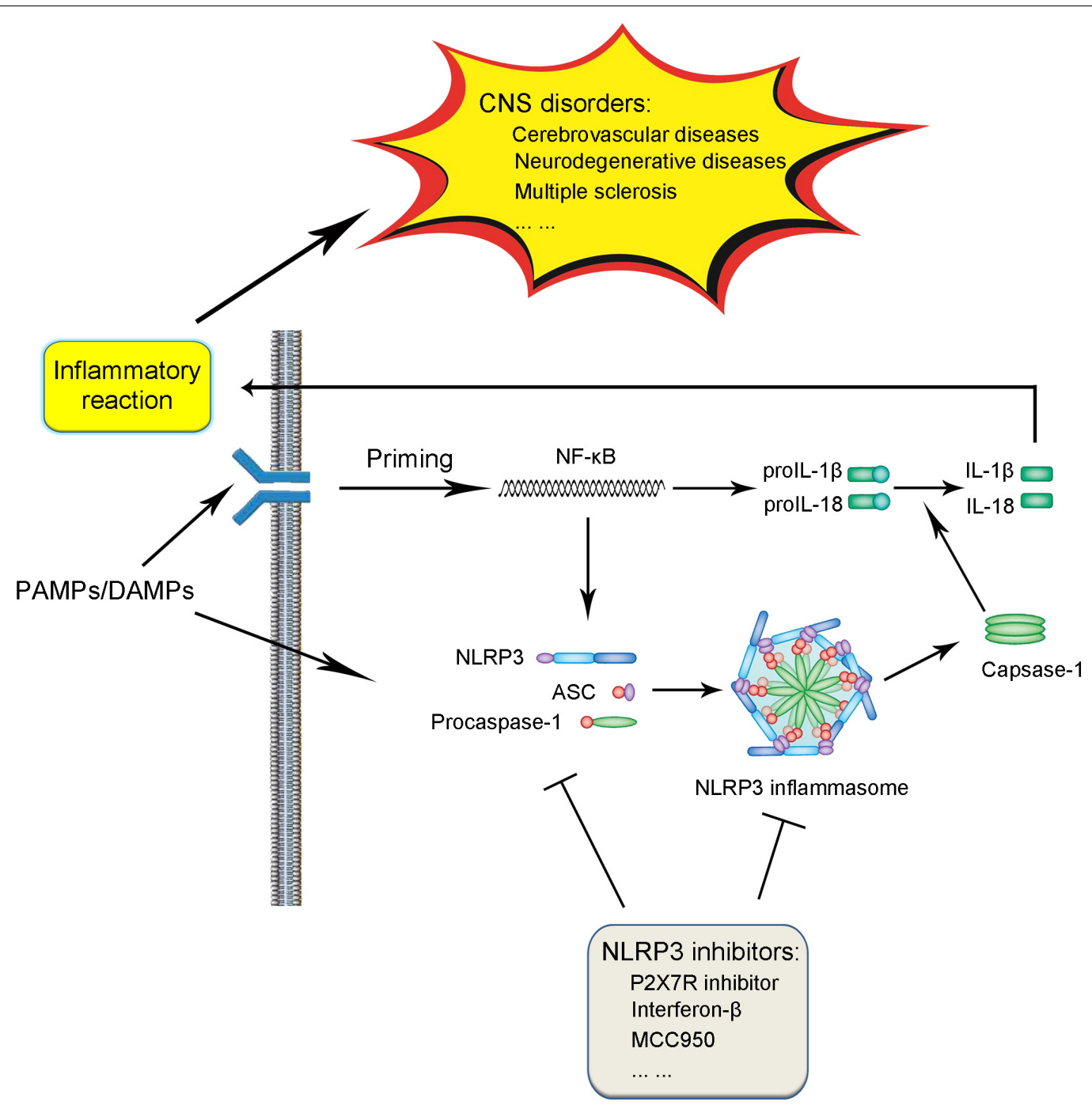

FIGURE 1 | Schematic illustration of biological process and signaling pathway of NLRP3 inflammasome and association with CNS disorders. Under the exposure of

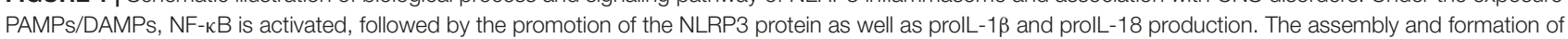
the NLRP3 inflammasome through the combination of NLRP3, ASC, and procaspase-1 triggered by further stimulation leads to the production of caspase-1, which catalyzes the transformation from prolL-1 $\beta$ and prolL-18 into IL-1 $\beta$ and IL-18. The production and release of IL-1 $\beta$ and IL-18 induces various forms of inflammatory reaction, thus contributing to the pathogenesis and progression of CNS diseases. The process of NLRP3 inflammasome activation can be blocked by several kinds of NLRP3 inflammasome inhibitors, which serve as potential therapeutic strategies against CNS disorders.

CNS disorders including cerebrovascular diseases (ischemic stroke and hemorrhagic stroke), neurodegenerative diseases (Alzheimer's disease, Huntington's disease, and Parkinson's disease), multiple sclerosis, depression as well as other CNS disorders. Furthermore, the pharmacological applications of the NLRP3 inflammasome inhibitors in the treatment of CNS diseases are included in the relative parts of discussion (Table 1).

\section{Cerebrovascular Diseases}

\section{NLRP3 Inflammasome in Cerebrovascular Diseases}

Cerebrovascular diseases refer to a group of pathological processes which result in the negative influence on cerebral vessels and circulation, thus leading to the subsequent damage of the brain tissues in function or structure. Cerebrovascular diseases are regarded as a group of major health problems for human beings worldwide, causing high morbidity, mortality as well as disability (Qureshi, 2004). It is widely acknowledged that there are two major types of cerebrovascular diseases, including ischemic and hemorrhagic stroke, with ischemic stroke referred to as decreased supply to certain brain regions and hemorrhagic stroke as bleeding into or around the brain tissue (Uchiyama, 2017; Gokcal et al., 2018).

The NLRP3 inflammasome has been reported to play a vital role in cerebrovascular diseases, and many signaling pathways mediated by the NLRP3 inflammasome are highly involved in the onset and progression of both ischemic and hemorrhagic stroke (Zhou et al., 2016; Barrington et al., 2017; Freeman et al., 2017; Ye et al., 2017; Fann et al., 2018; Nasoohi et al., 2018). For example, it 
TABLE 1 | Potential mechanisms of several NLRP3 inflammasome inhibitors in CNS diseases.

\begin{tabular}{|c|c|c|c|}
\hline CNS disease & NLRP3 inflammasome inhibitor & Potential mechanisms & Reference \\
\hline \multirow[t]{3}{*}{ Ischemic stroke } & Minocycline & Preventing microglia activation; Inhibiting Step 1 and 2 activation & Lu et al., 2016 \\
\hline & Sinomenin & Activating AMPK signaling & Qiu et al., 2016 \\
\hline & Nafamostat mesilate & Inhibiting NF-кB signaling & Li et al., 2016 \\
\hline \multirow[t]{3}{*}{ Hemorrhagic stroke } & P2X7R inhibitor & Inhibiting P2X7R & Feng et al., 2015 \\
\hline & Necrostatin-1 & Inhibiting RIP1-RIP3-DRP1 signaling & Zhou et al., 2017 \\
\hline & MicroRNA-223 & Downregulating NLRP3 expression & Yang et al., 2015 \\
\hline \multirow[t]{2}{*}{ Alzheimer's disease } & Edaravone & $\begin{array}{l}\text { Reducing mitochondria-derived ROS production; increasing SOD-2 } \\
\text { activity }\end{array}$ & Wang et al., 2017 \\
\hline & Depeptidyl vinyl sulfone & Decreasing microRNA-155 and microRNA-146a & Falcao et al., 2017 \\
\hline \multirow[t]{2}{*}{ Parkinson's disease } & MicroRNA-30e & Downregulating NLRP3, ASC and procaspase-1 expression & Li et al., 2018a \\
\hline & Tenuigenin & Downregulating ROS & Fan et al., 2017 \\
\hline Huntington's disease & P2X7R inhibitor & Inhibiting P2X7R & Sperlagh and IIles, 2014 \\
\hline \multirow[t]{4}{*}{ Multiple sclerosis/EAE } & Interferon- $\beta$ & Inducting STAT1 phosphorylation and IL-10 production & $\begin{array}{l}\text { Inoue et al., 2012; Inoue and } \\
\text { Shinohara, 2013b; Shao et al., } \\
\text { 2015; Inoue et al., } 2016\end{array}$ \\
\hline & DR $\alpha 1-m o u s e(m) M O G-35-55$ & Targeting on the MIF/CD74 pathway & Benedek et al., 2015, 2017 \\
\hline & $\mathrm{HU}-308$ & Activating CB2R; Inducing autophagy process & Shao et al., 2014 \\
\hline & PNU282987 & $\begin{array}{l}\text { Activating } \alpha 7 n A C h R ; \text { Downregulating NLRP3 and } \beta \text {-arrestin-1 } \\
\text { interaction }\end{array}$ & Ke et al., 2017 \\
\hline \multirow[t]{3}{*}{ Depression } & Salvianolic acide B & Promoting autophagy process & Jiang et al., 2017 \\
\hline & Astragaloside IV & Activating the PPAR $\gamma / N F-\kappa B / N L R P 3$ inflammasome axis & Song et al., 2018 \\
\hline & Electro-acupuncture & Reversing the increase of P2X7R & Yue et al., 2018 \\
\hline \multirow[t]{3}{*}{ Traumatic brain injury } & MCC950 & Suppressing the NLRP3 inflammasome priming process & Ismael et al., 2018 \\
\hline & Resveratrol & Promoting SIR1 signaling & Zou et al., 2018 \\
\hline & NOX2 inhibitor & Inhibiting NOX2 & Ma et al., 2017 \\
\hline
\end{tabular}

was previously reported that under the stimulation of brain injury or disturbance of glucose metabolism in CNS, the activation of the NLRP3 inflammasome was triggered by thioredoxininteracting protein (TXNIP), an endogenous inhibitor of the antioxidant thioredoxin (TRX) system, thus leading to the aggravation of brain tissue injury (Nasoohi et al., 2018). In addition, the purinergic $2 \mathrm{X} 7$ receptor (P2X7R)/NLRP3 pathway was shown to cause the enhancement of cerebral infarction volume and aggravation of neurological impairment through the triggering of the caspase- 3 dependent neuronal apoptosis in cerebral ischemia models (Ye et al., 2017). Another toxin, the lysophosphatidylcholine (LPC) might trigger the activation of the NLRP3 and NLRC4 inflammasome in microglia and astrocytes, thus contributing greatly to the enhancement of neuroinflammation (Freeman et al., 2017). Furthermore, Fann et al. (2018) demonstrated that both the NF- $\mathrm{KB}$ and mitogenactivated protein kinase (MAPK) signaling pathways could serve as important signaling pathways in regulating the expression and activation of the NLRP3 as well as NLRP1 inflammasomes in primary cortical neurons and brain tissues under ischemic conditions.

\section{Pharmacological Applications of NLRP3 Inflammasome Inhibitors in Cerebrovascular Diseases}

As reviewed by us previously, many promising inhibitors of the NLRP3 inflammasome have been demonstrated in the treatment of inflammatory and immune diseases, such as Type I interferon and interferon- $\beta$, small-molecule inhibitors and others like autophagy inducers (Shao et al., 2015). In cerebrovascular diseases, several agents taking advantage of inhibiting the NLRP3 inflammasome have been presented in the alleviation of ischemic or hemorrhagic stroke. For the treatment of ischemic stroke, it was reported that pretreatment of minocycline, a tetracycline antibiotic, significantly prevented the activation of microglia through the inhibition of the NLRP3 inflammasome in the two steps of activation, thus improving neurological disorder, reducing infarct volume and alleviating cerebral edema ( $\mathrm{Lu}$ et al., 2016). Additionally, a recent study demonstrated the neuroprotective effects of sinomenin, a kind of alkaloid derived from Sinomenium acutum, in mice cerebral artery occlusion (MCAO) ischemic models in vivo and oxygen glucose deprivation (OGD)-treated in vitro models (Qiu et al., 2016). Those protective effects were induced by the AMP-activated protein kinase (AMPK)-mediated inhibition of the NLRP3 inflammasome (Qiu et al., 2016). Furthermore, nafamostat mesilate, a wide-spectrum serine protease inhibitor, was shown to produce an antineuroinflammation effect in rat transient MCAO (tMCAO) mice as well as OGD in vitro models though the inhibition of the NFкB-mediated activation of the NLRP3 inflammasome (Li et al., 2016). For the treatment of hemorrhagic stroke, it was previously demonstrated that blue brilliant G (BBG), a selective P2X7R inhibitor, contributed to the alleviation of the intracerebral hemorrhage-induced inflammatory injury in rat models through the inhibition of the NLRP3 inflammasome activation as well as attenuation of NADPH oxidase 2 (NOX2) and inducible 
nitric oxide synthase (iNOS) production (Feng et al., 2015). In addition, necrostatin-1 was reported to attenuate the early brain injury after subarachnoid hemorrhage in rat models through the inhibition of the receptor-interacting protein (RIP)1-RIP3dynamin-related protein (DRP) 1 signaling pathway, thus leading to the suppression of the NLRP3 inflammasome activation (Zhou et al., 2017). Moreover, it was shown that microRNA-223 could inhibit the activation of the NLRP3 inflammasome through the downregulation of the NLRP3 protein expression, thus alleviating the brain injury after intracerebral hemorrhage (Yang et al., 2015). Taken together, although current studies have provided potentially effective agents for the treatment of cerebrovascular diseases targeting on the NLRP3 inflammasome, further studies are demanded for the exploration of their applications in clinic.

\section{Neurodegenerative Diseases NLRP3 Inflammasome in Neurodegenerative Diseases}

Neurodegenerative diseases refer to a group of disorders with progressive loss of structure or function of neurons and finally lead to neuronal death (Haass and Selkoe, 2007). There are three well-known neurodegenerative diseases worldwide, namely Alzheimer's, Parkinson's, and Huntington's diseases (Armstrong and Barker, 2001). One of the major causes of those three neurodegenerative diseases is regarded as the abnormal protein aggregation in neurons, including $\beta$-amyloid for the pathogenesis and progression of Alzheimer's disease, $\alpha$-synulein to form proteinaceous cytoplasmic Lewy bodies for Parkinson's disease and the aggregate-prone huntingtin protein for Huntington's disease (Chen and Tully, 2018; Clark et al., 2018; Kreutzer and Nowick, 2018; Matthes et al., 2018; Zhan et al., 2018; Zhang et al., 2018). Besides, the activation of the NLRP3 inflammasome also serves as a vital factor for the onset and development of neurodegenerative diseases (Ahmed et al., 2017; Fu et al., 2017; Li et al., 2017; Sarkar et al., 2017; Wu et al., 2017; Aminzadeh et al., 2018; Bai et al., 2018; Gong et al., 2018; Qi et al., 2018). It has been reported that the NLRP3 inflammasome activation significantly leads to the synaptic plasticity deficits in the pathogenesis of Alzheimer's disease (Qi et al., 2018). Several influential factors have been reported to lead to the activation of the NLRP3 inflammasome, including cathepsin $\mathrm{B}$, transient receptor potential melastatin 2 (TRPM2), mitochondrial impairment, microtubule-affinity regulating kinase $4(\mathrm{MARK} 4), \mathrm{K}^{+}, \mathrm{Ca}^{2+}$, and $\mathrm{Cl}^{-}$fluxes and so on (Ahmed et al., 2017; Fu et al., 2017; Li et al., 2017; Sarkar et al., 2017; Wu et al., 2017; Aminzadeh et al., 2018; Bai et al., 2018; Gong et al., 2018). As a result, inhibiting the NLRP3 inflammasome may serve as a potential and effective therapeutic strategy in the treatment of neurodegenerative diseases.

\section{Pharmacological Applications of NLRP3 Inflammasome Inhibitors in Neurodegenerative Diseases}

In the last few years, numerous studies have been conducted in the exploration of therapeutic pathways against neurodegenerative diseases through the inhibition of the NLRP3 inflammasome. For the studies of Alzheimer's disease, it was recently reported that edaravone, a recently shown oxidative stress suppressor, functioned in attenuating the $\beta$-amyloid-induced proinflammatory response in microglia (Wang et al., 2017). This effect was through the reduction of mitochondria-derived reactive oxygen species (ROS) production and increased manganese superoxide dismutase (SOD-2) activity, thus leading to the inhibition of the NLRP3 inflammasomemediated proinflammatory secretion (Wang et al., 2017). Furthermore, depeptidyl vinyl sulfone, a chemical agent, was demonstrated to suppress the high-mobility group box protein-1 (HMGB1)/NLRP3 inflammasome-related inflammation in $\beta$-amyloid-stimulated microglia, which was involved in the decrease of the two inflammation-related microRNA including microRNA-155 and microRNA-146a (Falcao et al., 2017). For the treatment of Parkinson's disease, it was reported that microRNA30e might serve as a potential treatment for Parkinson's disease therapeutics through the downregulation of the NLRP3 protein, ASC and procaspase-1 both in mRNA and protein levels, thus largely attenuating the NLRP3 inflammasome signaling (Li et al., 2018a). Furthermore, tenuigenin, a major active component derived from Polygala tenuifolia, was indicated to serve as a potential anti-inflammatory agent contributing to the treatment of Parkinson's disease (Fan et al., 2017). The anti-inflammatory effect was induced by the inhibition of the NLRP3 inflammasome activation through downregulation of ROS in microglia in mice Parkinson's disease models (Fan et al., 2017). For the treatment of Huntington's disease, although not much studies are available for the exploration of the therapeutic roles targeting on the NLRP3 inflammasome, there is one study demonstrating that pharmacological inhibition of the ATP-sensitive homomeric P2X7R contributes to the alleviation of Huntington's disease through the suppression of the NLRP3 inflammasome activation (Sperlagh and Illes, 2014). However, further efforts are demanded in the investigation of the NLRP3 inflammasome in the treatment of Huntington's disease.

\section{Multiple Sclerosis}

\section{NLRP3 Inflammasome in Multiple Sclerosis}

Multiple sclerosis is one of the most commonly diagnosed autoimmune diseases, characterized as demyelination and neurodegeneration, which also belongs to a neurodegenerative disorder (Shao et al., 2014; Rossi and Constantin, 2016). It is one of the most serious neurological diseases among young people, leading to progressive disabilities (Marck et al., 2016). The overreaction of the inflammatory and immune responses has been reported to contribute to the pathogenesis and progression of multiple sclerosis, in the occurrence of which was previously demonstrated to induce the highly expression of proinflammatory cytokines in microglia as well as CNS tissues in animal models of multiple sclerosis (Lieberknecht et al., 2017; Shao et al., 2017a; Lee et al., 2018a). The NLRP3 inflammasome has been reported to be involved in the development of multiple sclerosis through the secretion of IL-1 $\beta$ and IL-18 (Barclay and 
Shinohara, 2017). As a result, pharmacological inhibition of the NLRP3 inflammasome has been regarded as a potential target for the treatment of multiple sclerosis (Inoue and Shinohara, 2013a, 2015).

\section{Pharmacological Applications of NLRP3 Inflammasome Inhibitors in Multiple Sclerosis}

So far, several agents targeting on the inhibition of the NLRP3 inflammasome have been demonstrated to be effective in the treatment of multiple sclerosis or shown on the mice models of multiple sclerosis (experimental autoimmune encephalomyelitis, EAE). One of the most popular agents is interferon- $\beta$, which has already been applied in clinic as a first-line drug for the treatment of multiple sclerosis through the induction of the phosphorylation of STAT1 as well as IL-10 production (Inoue and Shinohara, 2013b; Shao et al., 2015). However, there were also studies demonstrating the limitation of the application of interferon- $\beta$ in the treatment of the multiple sclerosis or EAE, showing that interferon- $\beta$ therapy was effective against multiple sclerosis or EAE only in the NLRP3 inflammasomedependent EAE (Inoue et al., 2012; Inoue et al., 2016). In addition, Benedek et al. $(2015,2017)$ demonstrated that DR $\alpha 1$ mouse(m)MOG-35-55, a less immunogenic alternative to twodomain class II construct developed by their lab, significantly reversed the clinical and histological symptoms of EAE mice through the inhibition of the NLRP3 inflammasome targeting on the MIF/CD74 pathway. Moreover, previous studies in our lab also reported several effective NLRP3 inflammasome inhibitors in the treatment of EAE. We found that the administration of cannabinoid receptor 2 (CB2R) agonist HU308 significantly suppressed the activation of the NLRP3 inflammasome in microglia through the induction of autophagy process, thus producing an ameliorative effect on EAE (Shao et al., 2014). Furthermore, it was also reported by us that the activation of $\alpha 7$ nicotinic acetylcholine receptor ( $\alpha 7 \mathrm{nAChR})$ by PNU282987 largely suppressed the NLRP3 inflammasome in monocyte/macrophage system in EAE, thus leading to the alleviation of the severity of EAE (Ke et al., 2017). Those effects were mainly through the downregulation of the interaction between the NLRP3 protein and $\beta$-arrestin-1 in microglia (Ke et al., 2017).

\section{Depression}

\section{NLRP3 Inflammasome in Depression}

Another CNS disease highly related to the NLRP3 inflammasome is depression. Although it is regarded as a mental disorder, yet modern studies have demonstrated that the pathogenesis and progression of depression is involved in the overreaction of inflammatory and immune responses (Franklin et al., 2017). NLRP3-dependent caspase-1 activation was reported to be significantly implicated in the progression of systemic inflammation-induced depression triggered by LPS in mice depression model (Jeon et al., 2017). In addition, Lei et al. (2017) showed that neonatal inflammation or early-life inflammation stress could trigger the activation of the NLRP3 inflammasome through the up-regulation of the NLRP3 inflammasome-related proteins in the brain, thus leading to the increasing occurrence of anxiety-like behavior in adolescent rats. Consequently, targeting on the NLRP3 inflammasome might serve as a potential therapy in the alleviation of depression.

\section{Pharmacological Applications of NLRP3 Inflammasome Inhibitors in Depression}

So far, several agents have been reported to be effective in the alleviation of depression through the inhibition of the NLRP3 inflammasome. For example, it was recently demonstrated by Jiang et al. (2017) that salvianolic acid B, a natural compound extracted from Salvia miltiorrhize, contributed to the attenuation of depression in symptom through the promotion of the protective autophagy process, thus leading to the induction of the clearance of the NLRP3 protein. Astragaloside IV, an active component purified from Astragalus membranaceus (Fisch) Bge, attenuated the neuroinflammation-induced depressivelike behaviors in mice through the PPAR $\gamma / \mathrm{NF}-\kappa \mathrm{B} / \mathrm{NLRP} 3$ inflammasome axis (Song et al., 2018). In addition, other agents targeting on the inhibition of the NLRP3 inflammasome were reported in animal depression models, although the specific mechanisms remained unclear (Cao et al., 2017; Liu and Liu, 2017). Besides, it was recently reported that electro-acupuncture, one of the popular techniques of traditional Chinese medicine, contributed to the alleviation of the chronic unpredictable stress-induced depression and anxiety-like behaviors through the inhibition of the NLRP3 inflammasome activation, which was mediated by reversing the increase of P2X7R (Yue et al., 2018).

\section{Others}

Besides those popular groups of CNS disorders discussed above, targeting on the inhibition of the NLRP3 inflammasome has been reported to be effective in other kinds of CNS diseases. For example, traumatic brain injury, a major cause of death and disability all over the world especially among children and teenagers, was considered to lead to the neuropathological conditions, which consequently cause the initiation of the production of the proinflammatory cytokines (Mortezaee et al., 2017). The NLRP3 inflammasome has been demonstrated to play a pivotal role in the development of traumatic brain injury through several kinds of mechanisms including RIP3related pathway, activation of cortical microglia and so on (Lee et al., 2018b; Liu et al., 2018). As a result, inhibiting the NLRP3 inflammasome in CNS serves as a potential and effective pathway for the attenuation of the development of traumatic brain injury. For example, MCC950, a selective NLRP3 inflammasome inhibitor, was reported to alleviate the severity of traumatic brain injury in experimental animal models through the suppression of the NLRP3 inflammasome priming process (Ismael et al., 2018). In addition, resveratrol, a natural autophagy inducer, was demonstrated to function in the inhibition of the NLRP3 inflammasome in cerebral cortex through the promotion of sirtuin 1 (SIR1) signaling pathway (Zou et al., 2018). Furthermore, NOX2 was shown to contribute to the progression of traumatic brain injury, and the administration of the NOX2 inhibitors led to the a neuroprotective effect 
through the suppression of the NLRP3 inflammasome (Ma et al., 2017).

\section{CONCLUDING REMARKS}

All in all, recent studies have demonstrated the important roles of the NLRP3 inflammasome in the pathogenesis and progression of various kinds of CNS diseases (Figure 1). So far, we are lucky to have many kinds of inhibitors of the NLRP3 inflammasome activation developed, which have already been shown to be effective in the alleviation of certain CNS diseases in patients or animal models. However, because of the limitations of modern studies, there is still a long way to go for their successful application in clinic and get rid of the side effects. Further efforts are demanded to develop potential and effective therapeutic

\section{REFERENCES}

Ahmed, M. E., Iyer, S., Thangavel, R., Kempuraj, D., Selvakumar, G. P., Raikwar, S. P., et al. (2017). Co-Localization of glia maturation factor with NLRP3 inflammasome and autophagosome markers in human alzheimer's disease brain. J. Alzheimers. Dis. 60, 1143-1160. doi: 10.3233/JAD-170634

Aminzadeh, M., Roghani, M., Sarfallah, A., and Riazi, G. H. (2018). TRPM2 dependence of ROS-induced NLRP3 activation in Alzheimer's disease. Int. Immunopharmacol. 54, 78-85. doi: 10.1016/j.intimp.2017.10.024

Anuncibay-Soto, B., Perez-Rodriguez, D., Santos-Galdiano, M., Font-Belmonte, E., Ugidos, I. F., Gonzalez-Rodriguez, P., et al. (2018). Salubrinal and robenacoxib treatment after global cerebral ischemia. Exploring the interactions between ER stress and inflammation. Biochem. Pharmacol. 151, 26-37. doi: 10.1016/j.bcp. 2018.02.029

Armstrong, R. J., and Barker, R. A. (2001). Neurodegeneration: a failure of neuroregeneration? Lancet 358, 1174-1176. doi: 10.1016/S0140-6736(01) 06260-2

Awad, F., Assrawi, E., Louvrier, C., Jumeau, C., Georgin-Lavialle, S., Grateau, G., et al. (2018). Inflammasome biology, molecular pathology and therapeutic implications. Pharmacol. Ther. 187:133-149. doi: 10.1016/j.pharmthera.2018. 02.011

Bai, H., Yang, B., Yu, W., Xiao, Y., Yu, D., and Zhang, Q. (2018). Cathepsin B links oxidative stress to the activation of NLRP3 inflammasome. Exp. Cell Res. 362, 180-187. doi: 10.1016/j.yexcr.2017.11.015

Barclay, W., and Shinohara, M. L. (2017). Inflammasome activation in multiple sclerosis and experimental autoimmune encephalomyelitis (EAE). Brain Pathol. 27, 213-219. doi: 10.1111/bpa.12477

Barrington, J., Lemarchand, E., and Allan, S. M. (2017). A brain in flame; do inflammasomes and pyroptosis influence stroke pathology? Brain Pathol. 27, 205-212. doi: 10.1111/bpa.12476

Benedek, G., Chaudhary, P., Meza-Romero, R., Calkins, E., Kent, G., Offner, H., et al. (2017). Sex-dependent treatment of chronic EAE with partial MHC class II constructs. J. Neuroinflammation 14:100. doi: 10.1186/s12974-0170873-y

Benedek, G., Meza-Romero, R., Jordan, K., Keenlyside, L., Offner, H., and Vandenbark, A. A. (2015). HLA-DRalpha1-mMOG-35-55 treatment of experimental autoimmune encephalomyelitis reduces CNS inflammation, enhances M2 macrophage frequency, and promotes neuroprotection. J. Neuroinflammation 12:123. doi: 10.1186/s12974-015-0342-4

Birnbaum, Y., Bajaj, M., Yang, H. C., and Ye, Y. (2018). Combined SGLT2 and DPP4 inhibition reduces the activation of the Nlrp3/ASC inflammasome and attenuates the development of diabetic nephropathy in mice with type 2 diabetes. Cardiovasc. Drugs Ther. 32, 135-145. doi: 10.1007/s10557-0186778-x

Cacabelos, R., Torrellas, C., Fernandez-Novoa, L., and Lopez-Munoz, F. (2016). Histamine and immune biomarkers in CNS disorders. Mediat. Inflamm. 2016:1924603. doi: $10.1155 / 2016 / 1924603$ strategies against CNS diseases taking advantage of the NLRP3 inflammasome inhibition.

\section{AUTHOR CONTRIBUTIONS}

B-ZS retrieved concerned literatures and wrote the manuscript. QC designed the table. CL revised the manuscript. All the authors agreed to be accountable for the content of the work.

\section{FUNDING}

This work was supported by two grants from the National Natural Science Foundation of China (81670260 and 81871596) and a grant from Shanghai Pujiang Program (18PJD062).

Cao, C., Su, M., and Zhou, F. (2017). Mangiferin inhibits hippocampal NLRP3 inflammasome and exerts antidepressant effects in a chronic mild stress mice model. Behav. Pharmacol. 28, 356-364. doi: 10.1097/FBP.0000000000000305

Chen, A. Y., and Tully, T. (2018). A stress-enhanced model for discovery of disease-modifying gene: ecel-suppresses the toxicity of alpha-synuclein A30P. Neurobiol. Dis. 114, 153-163. doi: 10.1016/j.nbd.2018.03.003

Chen, L., You, Q., Hu, L., Gao, J., Meng, Q., Liu, W., et al. (2017). The antioxidant procyanidin reduces reactive oxygen species signaling in macrophages and ameliorates experimental colitis in mice. Front. Immunol. 8:1910. doi: 10.3389/ fimmu.2017.01910

Chen, M., Lu, X., Lu, C., Shen, N., Jiang, Y., Chen, M., et al. (2018). Soluble uric acid increases PDZK1 and ABCG2 expression in human intestinal cell lines via the TLR4-NLRP3 inflammasome and PI3K/Akt signaling pathway. Arthritis Res. Ther. 20:20. doi: 10.1186/s13075-018-1512-4

Chu, L. H., Indramohan, M., Ratsimandresy, R. A., Gangopadhyay, A., Morris, E. P., Monack, D. M., et al. (2018). The oxidized phospholipid oxPAPC protects from septic shock by targeting the non-canonical inflammasome in macrophages. Nat. Commun. 9:996. doi: 10.1038/s41467-018-03409-3

Clark, L. R., Berman, S. E., Norton, D., Koscik, R. L., Jonaitis, E., Blennow, K., et al. (2018). Age-accelerated cognitive decline in asymptomatic adults with CSF beta-amyloid. Neurology 90:e1306-e1315. doi: 10.1212/WNL. 0000000000005291

Correa-da-Silva, F., Pereira, J. A. S., De Aguiar, C. F., and De Moraes-Vieira, P. M. M. (2017). Mitoimmunity-when mitochondria dictates macrophage function. Cell Biol Int. 42, 651-655. doi: 10.1002/cbin.10921

de Rivero Vaccari, J. P., Dietrich, W. D., and Keane, R. W. (2014). Activation and regulation of cellular inflammasomes: gaps in our knowledge for central nervous system injury. J. Cereb. Blood Flow Metab. 34, 369-375 doi: 10.1038/ jcbfm.2013.227

Di Virgilio, F., Dal Ben, D., Sarti, A. C., Giuliani, A. L., and Falzoni, S. (2017). The P2X7 receptor in infection and inflammation. Immunity 47, 15-31. doi: 10.1016/j.immuni.2017.06.020

Du, S. Q., Wang, X. R., Zhu, W., Ye, Y., Yang, J. W., Ma, S. M., et al. (2018). Acupuncture inhibits TXNIP-associated oxidative stress and inflammation to attenuate cognitive impairment in vascular dementia rats. CNS Neurosci. Ther. 24, 39-46. doi: 10.1111/cns.12773

Duncan, J. A., and Canna, S. W. (2018). The NLRC4 inflammasome. Immunol. Rev. 281, 115-123. doi: 10.1111/imr.12607

Falcao, A. S., Carvalho, L. A., Lidonio, G., Vaz, A. R., Lucas, S. D., Moreira, R., et al. (2017). Dipeptidyl vinyl sulfone as a novel chemical tool to inhibit HMGB1/NLRP3-inflammasome and inflamma-mirs in abetamediated microglial inflammation. ACS Chem. Neurosci. 8, 89-99. doi: 10.1021/ acschemneuro.6b00250

Fan, Z., Liang, Z., Yang, H., Pan, Y., Zheng, Y., and Wang, X. (2017). Tenuigenin protects dopaminergic neurons from inflammation via suppressing NLRP3 inflammasome activation in microglia. J. Neuroinflammation 14:256. doi: 10.1186/s12974-017-1036-x 
Fann, D. Y., Lim, Y. A., Cheng, Y. L., Lok, K. Z., Chunduri, P., Baik, S. H., et al. (2018). Evidence that NF-kappaB and MAPK signaling promotes NLRP inflammasome activation in neurons following ischemic stroke. Mol. Neurobiol. 55, 1082-1096. doi: 10.1007/s12035-017-0394-9

Feng, L., Chen, Y., Ding, R., Fu, Z., Yang, S., Deng, X., et al. (2015). P2X7R blockade prevents NLRP3 inflammasome activation and brain injury in a rat model of intracerebral hemorrhage: involvement of peroxynitrite. J. Neuroinflammation 12:190. doi: 10.1186/s12974-015-0409-2

Franklin, T. C., Xu, C., and Duman, R. S. (2017). Depression and sterile inflammation: essential role of danger associated molecular patterns. Brain Behav Immun. 72, 2-13. doi: 10.1016/j.bbi.2017.10.025

Freeman, L., Guo, H., David, C. N., Brickey, W. J., Jha, S., and Ting, J. P. (2017). NLR members NLRC4 and NLRP3 mediate sterile inflammasome activation in microglia and astrocytes. J. Exp. Med. 214, 1351-1370. doi: 10.1084/jem. 20150237

Fu, W., Vukojevic, V., Patel, A., Soudy, R., Mactavish, D., Westaway, D., et al. (2017). Role of microglial amylin receptors in mediating beta amyloid (Abeta)induced inflammation. J. Neuroinflammation 14:199. doi: 10.1186/s12974-0170972-9

Gambin, Y., Giles, N., O'carroll, A., Polinkovsky, M., Hunter, D., and Sierecki, E. (2018). Single-molecule fluorescence reveals the oligomerization and folding steps driving the prion-like behavior of ASC. J. Mol. Biol. 430, 491-508. doi: 10.1016/j.jmb.2017.12.013

Gokcal, E., Pasi, M., Fisher, M., and Gurol, M. E. (2018). Atrial fibrillation for the neurologist: preventing both ischemic and hemorrhagic strokes. Curr. Neurol. Neurosci. Rep. 18:6. doi: 10.1007/s11910-018-0813-y

Gong, T., Yang, Y., Jin, T., Jiang, W., and Zhou, R. (2018). Orchestration of NLRP3 inflammasome activation by ion fluxes. Trends Immunol. 39, 393-406. doi: 10.1016/j.it.2018.01.009

Haass, C., and Selkoe, D. J. (2007). Soluble protein oligomers in neurodegeneration: lessons from the Alzheimer's amyloid beta-peptide. Nat. Rev. Mol. Cell Biol. 8, 101-112. doi: 10.1038/nrm2101

He, Z., Chen, J., An, S., Dong, X., Yu, J., Zhang, S., et al. (2018). The NLRP3 inflammasome activation mediates Zika virus associated inflammation. J. Infect. Dis. 217, 1942-1951. doi: 10.1093/infdis/jiy129

Heo, M. J., Kim, T. H., You, J. S., Blaya, D., Sancho-Bru, P., and Kim, S. G. (2018). Alcohol dysregulates miR-148a in hepatocytes through FoxO1, facilitating pyroptosis via TXNIP overexpression. Gut doi: 10.1136/gutjnl-2017-315123 [Epub ahead of print].

Ho, S. C., and Chang, Y. H. (2018). Comparison of inhibitory capacities of 6-, 8and 10-gingerols/shogaols on the canonical NLRP3 inflammasome-mediated IL-1beta secretion. Molecules 23:E466. doi: 10.3390/molecules23020466

Inoue, M., Chen, P. H., Siecinski, S., Li, Q. J., Liu, C., Steinman, L., et al. (2016). An interferon-beta-resistant and NLRP3 inflammasome-independent subtype of EAE with neuronal damage. Nat. Neurosci. 19, 1599-1609. doi: 10.1038/nn.4421

Inoue, M., and Shinohara, M. L. (2013a). NLRP3 inflammasome and MS/EAE. Autoimmune Dis. 2013:859145. doi: 10.1155/2013/859145

Inoue, M., and Shinohara, M. L. (2013b). The role of interferon-beta in the treatment of multiple sclerosis and experimental autoimmune encephalomyelitis - in the perspective of inflammasomes. Immunology 139, 11-18. doi: 10.1111/imm.12081

Inoue, M., and Shinohara, M. L. (2015). NLRP3 inflammasome and multiple sclerosis/EAE. Nihon Rinsho 73(Suppl. 7), 835-841.

Inoue, M., Williams, K. L., Oliver, T., Vandenabeele, P., Rajan, J. V., Miao, E. A., et al. (2012). Interferon-beta therapy against EAE is effective only when development of the disease depends on the NLRP3 inflammasome. Sci. Signal. 5:ra38. doi: 10.1126/scisignal.2002767

Ismael, S., Nasoohi, S., and Ishrat, T. (2018). MCC950, the selective NLRP3 inflammasome inhibitor protects mice against traumatic brain injury. J. Neurotrauma. 35, 1294-1303. doi: 10.1089/neu.2017.5344

Ito, M., Shichita, T., Okada, M., Komine, R., Noguchi, Y., Yoshimura, A., et al. (2015). Bruton's tyrosine kinase is essential for NLRP3 inflammasome activation and contributes to ischaemic brain injury. Nat. Commun. 6:7360. doi: $10.1038 /$ ncomms 8360

Jeon, S. A., Lee, E., Hwang, I., Han, B., Park, S., Son, S., et al. (2017). NLRP3 inflammasome contributes to Lipopolysaccharide-induced depressive-like behaviors via indoleamine 2,3-dioxygenase induction. Int. J. Neuropsychopharmacol. 20, 896-906. doi: 10.1093/ijnp/pyx065
Jiang, P., Guo, Y., Dang, R., Yang, M., Liao, D., Li, H., et al. (2017). Salvianolic acid B protects against lipopolysaccharide-induced behavioral deficits and neuroinflammatory response: involvement of autophagy and NLRP3 inflammasome. J. Neuroinflammation 14:239. doi: 10.1186/s12974-0171013-4

Kammoun, H. L., Allen, T. L., Henstridge, D. C., Barre, S., Coll, R. C., Lancaster, G. I., et al. (2018). Evidence against a role for NLRP3-driven islet inflammation in db/db mice. Mol Metab. 10, 66-73. doi: 10.1016/j.molmet.2018.02.001

Ke, P., Shao, B. Z., Xu, Z. Q., Chen, X. W., Wei, W., and Liu, C. (2017). Activating alpha7 nicotinic acetylcholine receptor inhibits NLRP3 inflammasome through regulation of beta-arrestin-1. CNS Neurosci. Ther. 23, 875-884. doi: 10.1111/ cns. 12758

Kosmidou, C., Efstathiou, N. E., Hoang, M. V., Notomi, S., Konstantinou, E. K., Hirano, M., et al. (2018). Issues with the specificity of immunological reagents for NLRP3: implications for age-related macular degeneration. Sci. Rep. 8:461. doi: 10.1038/s41598-017-17634-1

Kreutzer, A. G., and Nowick, J. S. (2018). Elucidating the structures of amyloid oligomers with macrocyclic beta-hairpin peptides: insights into alzheimer's disease and other amyloid diseases. Acc. Chem. Res. 51, 706-718 doi: 10.1021/ acs.accounts.7b00554

Kuemmerle-Deschner, J. B., Ozen, S., Tyrrell, P. N., Kone-Paut, I., GoldbachMansky, R., Lachmann, H., et al. (2017). Diagnostic criteria for cryopyrinassociated periodic syndrome (CAPS). Ann. Rheum. Dis. 76, 942-947. doi: 10.1136/annrheumdis-2016-209686

Landmann, E. C., and Walker, U. A. (2017). Pharmacological treatment options for cryopyrin-associated periodic syndromes. Expert. Rev. Clin. Pharmacol. 10, 855-864. doi: 10.1080/17512433.2017.1338946

Lee, C. H., Jeon, S. J., Cho, K. S., Moon, E., Sapkota, A., Jun, H. S., et al. (2018a). Activation of glucagon-like peptide-1 receptor promotes neuroprotection in experimental autoimmune encephalomyelitis by reducing neuroinflammatory responses. Mol. Neurobiol. 55, 3007-3020. doi: 10.1007/s12035-017-0550-2

Lee, S. W., Gajavelli, S., Spurlock, M. S., Andreoni, C., De Rivero Vaccari, J. P., Bullock, M. R., et al. (2018b). Microglial inflammasome activation in penetrating ballistic-like brain injury. J. Neurotrauma. 35, 1681-1693. doi: 10.1089/neu.2017.5530

Leentjens, J., Bekkering, S., Joosten, L. A. B., Netea, M. G., Burgner, D. P., and Riksen, N. P. (2018). Trained innate immunity as a novel mechanism linking infection and the development of atherosclerosis. Circ. Res. 122, 664-669. doi: 10.1161/CIRCRESAHA.117.312465

Lei, Y., Chen, C. J., Yan, X. X., Li, Z., and Deng, X. H. (2017). Earlylife lipopolysaccharide exposure potentiates forebrain expression of NLRP3 inflammasome proteins and anxiety-like behavior in adolescent rats. Brain Res. 1671, 43-54. doi: 10.1016/j.brainres.2017.06.014

Lewis, G. J., Massey, D. C., Zhang, H., Bredin, F., Tremelling, M., Lee, J. C., et al. (2011). Genetic association between NLRP3 variants and Crohn's disease does not replicate in a large UK panel. Inflamm. Bowel Dis. 17, 1387-1391. doi: 10.1002/ibd.21499

Li, C., Wang, J., Fang, Y., Liu, Y., Chen, T., Sun, H., et al. (2016). Nafamostat mesilate improves function recovery after stroke by inhibiting neuroinflammation in rats. Brain Behav. Immun. 56, 230-245. doi: 10.1016/j. bbi.2016.03.019

Li, D., Yang, H., Ma, J., Luo, S., Chen, S., and Gu, Q. (2018a). MicroRNA30e regulates neuroinflammation in MPTP model of Parkinson's disease by targeting Nlrp3. Hum. Cell 31, 106-115. doi: 10.1007/s13577-017-0187-5

Li, X., Yan, X., Wang, Y., Wang, J., Zhou, F., Wang, H., et al. (2018b). NLRP3 inflammasome inhibition attenuates silica-induced epithelial to mesenchymal transition (EMT) in human bronchial epithelial cells. Exp. Cell Res. 362, 489-497. doi: 10.1016/j.yexcr.2017.12.013

Li, X., Thome, S., Ma, X., Amrute-Nayak, M., Finigan, A., Kitt, L., et al. (2017). MARK4 regulates NLRP3 positioning and inflammasome activation through a microtubule-dependent mechanism. Nat. Commun. 8:15986. doi: 10.1038/ ncomms 15986

Lieberknecht, V., Junqueira, S. C., Cunha, M. P., Barbosa, T. A., De Souza, L. F., Coelho, I. S., et al. (2017). Pramipexole, a dopamine D2/D3 receptor-preferring agonist, prevents experimental autoimmune encephalomyelitis development in mice. Mol. Neurobiol. 54, 1033-1045. doi: 10.1007/s12035-016-9717-5

Liu, X., and Liu, C. (2017). Baicalin ameliorates chronic unpredictable mild stressinduced depressive behavior: Involving the inhibition of NLRP3 inflammasome 
activation in rat prefrontal cortex. Int. Immunopharmacol. 48, 30-34. doi: 10. 1016/j.intimp.2017.04.019

Liu, Z. M., Chen, Q. X., Chen, Z. B., Tian, D. F., Li, M. C., Wang, J. M., et al. (2018). RIP3 deficiency protects against traumatic brain injury (TBI) through suppressing oxidative stress, inflammation and apoptosis: dependent on AMPK pathway. Biochem Biophys Res Commun. 499, 112-119 doi: 10.1016/j.bbrc.2018. 02.150

Lu, Y., Xiao, G., and Luo, W. (2016). Minocycline suppresses NLRP3 inflammasome activation in experimental ischemic stroke. Neuroimmunomodulation 23, 230-238. doi: 10.1159/000452172

Lugrin, J., and Martinon, F. (2018). The AIM2 inflammasome: sensor of pathogens and cellular perturbations. Immunol. Rev. 281, 99-114. doi: 10.1111/imr.12618

Ma, M. W., Wang, J., Dhandapani, K. M., and Brann, D. W. (2017). NADPH oxidase 2 regulates NLRP3 inflammasome activation in the brain after traumatic brain injury. Oxid. Med. Cell Longev. 2017:6057609. doi: 10.1155/ 2017/6057609

Malik, A., and Kanneganti, T. D. (2017). Inflammasome activation and assembly at a glance. J. Cell Sci. 130, 3955-3963. doi: 10.1242/jcs.207365

Mangan, M.S., and Latz, E. (2018). TAK1ng control: TAK1 restrains NLRP3 activation. J. Exp. Med. 215, 1007-1008. doi: 10.1084/jem.20180282

Marck, C. H., Neate, S. L., Taylor, K. L., Weiland, T. J., and Jelinek, G. A. (2016). Prevalence of comorbidities, overweight and obesity in an international sample of people with multiple sclerosis and associations with modifiable lifestyle factors. PLoS One 11:e0148573. doi: 10.1371/journal.pone.0148573

Martinez, G. J., Celermajer, D. S., and Patel, S. (2018). The NLRP3 inflammasome and the emerging role of colchicine to inhibit atherosclerosis-associated inflammation. Atherosclerosis 269, 262-271. doi: 10.1016/j.atherosclerosis. 2017. 12.027

Matthes, F., Massari, S., Bochicchio, A., Schorpp, K., Schilling, J., Weber, S., et al. (2018). Reducing mutant huntingtin protein expression in living cells by a newly identified RNA CAG binder. ACS Chem. Neurosci. 9, 1399-1408 doi: 10.1021/acschemneuro.8b00027

McAllister, M. J., Chemaly, M., Eakin, A. J., Gibson, D. S., and Mcgilligan, V. E. (2018). NLRP3 as a potentially novel biomarker for the management of osteoarthritis. Osteoarthr. Cartil. 26: 612-619. doi: 10.1016/j.joca.2018.02.901

Meng, Y., Pan, M. X., Zheng, B. J., Chen, Y., Li, W., Yang, Q. J., et al. (2018). Autophagy attenuates angiotensin II-induced pulmonary fibrosis by inhibiting redox imbalance-mediated NLRP3 inflammasome activation. Antioxid. Redox Signal. doi: 10.1089/ars.2017.7261 [Epub ahead of print].

Mortezaee, K., Khanlarkhani, N., Beyer, C., and Zendedel, A. (2017). Inflammasome: its role in traumatic brain and spinal cord injury. J. Cell. Physiol. 223, 5160-5169 doi: 10.1002/jcp.26287

Nasoohi, S., Ismael, S., and Ishrat, T. (2018). Thioredoxin-interacting protein (TXNIP) in cerebrovascular and neurodegenerative diseases: regulation and implication. Mol Neurobiol. doi: 10.1007/s12035-018-0917-z [Epub ahead of print].

Ozaki, E., Campbell, M., and Doyle, S. L. (2015). Targeting the NLRP3 inflammasome in chronic inflammatory diseases: current perspectives. J. Inflamm. Res. 8, 15-27. doi: 10.2147/JIR.S51250

Place, D. E., and Kanneganti, T. D. (2018). Recent advances in inflammasome biology. Curr. Opin. Immunol. 50, 32-38. doi: 10.1016/j.coi.2017.10.011

Qi, Y., Klyubin, I., Cuello, A. C., and Rowan, M. J. (2018). NLRP3-dependent synaptic plasticity deficit in an Alzheimer's disease amyloidosis model in vivo. Neurobiol. Dis. 114, 24-30. doi: 10.1016/j.nbd.2018.02.016

Qiu, J., Wang, M., Zhang, J., Cai, Q., Lu, D., Li, Y., et al. (2016). The neuroprotection of sinomenine against ischemic stroke in mice by suppressing NLRP3 inflammasome via AMPK signaling. Int. Immunopharmacol. 40, 492-500. doi: 10.1016/j.intimp.2016.09.024

Qureshi, A. I. (2004). Endovascular treatment of cerebrovascular diseases and intracranial neoplasms. Lancet 363, 804-813. doi: 10.1016/S0140-6736(04) 15697-3

Rayasam, A., Hsu, M., Kijak, J. A., Kissel, L., Hernandez, G., Sandor, M., et al. (2018). Immune responses in stroke: how the immune system contributes to damage and healing after stroke and how this knowledge could be translated to better cures? Immunology 154, 363-376. doi: 10.1111/imm.12918

Rossi, B., and Constantin, G. (2016). Live imaging of immune responses in experimental models of multiple sclerosis. Front. Immunol. 7:506. doi: 10.3389/ fimmu.2016.00506
Sano, S., Oshima, K., Wang, Y., Maclauchlan, S., Katanasaka, Y., Sano, M., et al. (2018). Tet2-mediated clonal hematopoiesis accelerates heart failure through a mechanism involving the IL-1beta/NLRP3 inflammasome. J. Am. Coll. Cardiol. 71, 875-886. doi: 10.1016/j.jacc.2017.12.037

Sarkar, S., Malovic, E., Harishchandra, D. S., Ghaisas, S., Panicker, N., Charli, A., et al. (2017). Mitochondrial impairment in microglia amplifies NLRP3 inflammasome proinflammatory signaling in cell culture and animal models of Parkinson's disease. NPJ Parkinsons Dis. 3:30. doi: 10.1038/s41531-0170032-2

Sekerdag, E., Solaroglu, I., and Gursoy-Ozdemir, Y. (2018). Cell death mechanisms in stroke and novel molecular and cellular treatment options. Curr. Neuropharmacol. doi: 10.2174/1570159X16666180302115544 [Epub ahead of print].

Shao, B. Z., Ke, P., Xu, Z. Q., Wei, W., Cheng, M. H., Han, B. Z., et al. (2017a). Autophagy plays an important role in anti-inflammatory mechanisms stimulated by alpha7 nicotinic acetylcholine receptor. Front. Immunol. 8:553. doi: 10.3389/fimmu.2017.00553

Shao, B. Z., Wei, W., Ke, P., Xu, Z. Q., Zhou, J. X., and Liu, C. (2014). Activating cannabinoid receptor 2 alleviates pathogenesis of experimental autoimmune encephalomyelitis via activation of autophagy and inhibiting NLRP3 inflammasome. CNS Neurosci. Ther. 20, 1021-1028. doi: 10.1111/cns. 12349

Shao, B. Z., Xu, Z. Q., Han, B. Z., Su, D. F., and Liu, C. (2015). NLRP3 inflammasome and its inhibitors: a review. Front. Pharmacol. 6:262. doi: 10.3389/fphar.2015.00262

Shao, Q. H., Zhang, X. L., Yang, P. F., Yuan, Y. H., and Chen, N. H. (2017b). Amyloidogenic proteins associated with neurodegenerative diseases activate the NLRP3 inflammasome. Int. Immunopharmacol. 49, 155-160. doi: 10.1016/j. intimp.2017.05.027

Sharma, A., Tate, M., Mathew, G., Vince, J. E., Ritchie, R. H., and De Haan, J. B. (2018). Oxidative stress and NLRP3-inflammasome activity as significant drivers of diabetic cardiovascular complications: therapeutic implications. Front. Physiol. 9:114. doi: 10.3389/fphys.2018.00114

Shen, H. H., Yang, Y. X., Meng, X., Luo, X. Y., Li, X. M., Shuai, Z. W., et al. (2018). NLRP3: a promising therapeutic target for autoimmune diseases. Autoimmun. Rev. 17, 694-702. doi: 10.1016/j.autrev.2018.01.020

Shibata, K. (2018). Historical aspects of studies on roles of the inflammasome in the pathogenesis of periodontal diseases. Mol. Oral Microbiol. 33, 203-211. doi: $10.1111 /$ omi. 12217

Singh, S., and Jha, S. (2018). NLRs as helpline in the brain: mechanisms and therapeutic implications. Mol Neurobiol. doi: 10.1007/s12035-018-0957-4 [Epub ahead of print].

Song, L., Pei, L., Yao, S., Wu, Y., and Shang, Y. (2017). NLRP3 inflammasome in neurological diseases, from functions to therapies. Front. Cell. Neurosci. 11:63. doi: $10.3389 /$ fncel.2017.00063

Song, M. T., Ruan, J., Zhang, R. Y., Deng, J., Ma, Z. Q., and Ma, S. P. (2018). Astragaloside IV ameliorates neuroinflammation-induced depressivelike behaviors in mice via the PPARgamma/NF-kappaB/NLRP3 inflammasome axis. Acta Pharmacol. Sin. doi: 10.1038/aps.2017.208 [Epub ahead of print].

Speeckaert, R., Lambert, J., Grine, L., Van Gele, M., De Schepper, S., and Van Geel, N. (2016). The many faces of interleukin-17 in inflammatory skin diseases. Br. J. Dermatol. 175, 892-901. doi: 10.1111/bjd.14703

Sperlagh, B., and Illes, P. (2014). P2X7 receptor: an emerging target in central nervous system diseases. Trends Pharmacol. Sci. 35, 537-547. doi: 10.1016/j.tips. 2014.08.002

Stephenson, J., Nutma, E., Van Der Valk, P., and Amor, S. (2018). Inflammation in CNS neurodegenerative diseases. Immunology 154, 204-219. doi: 10.1111/imm. 12922

Suslov, A., Wieland, S., and Menne, S. (2018). Modulators of innate immunity as novel therapeutics for treatment of chronic hepatitis B. Curr. Opin. Virol. 30, 9-17. doi: 10.1016/j.coviro.2018.01.008

Sutterwala, F. S., Haasken, S., and Cassel, S. L. (2014). Mechanism of NLRP3 inflammasome activation. Ann. N. Y. Acad. Sci. 1319, 82-95. doi: 10.1111/nyas. 12458

Thaiss, C. A., Zmora, N., Levy, M., and Elinav, E. (2016). The microbiome and innate immunity. Nature 535, 65-74. doi: 10.1038/nature 18847

Uchiyama, S. (2017). Aspirin for primary stroke prevention in elderly patients with vascular risk factors. J. Gen. Fam. Med. 18, 331-335. doi: 10.1002/jgf2.102 
Wang, H. M., Zhang, T., Huang, J. K., Xiang, J. Y., Chen, J. J., Fu, J. L., et al. (2017). Edaravone attenuates the proinflammatory response in amyloid-beta-treated microglia by inhibiting NLRP3 inflammasome-mediated IL-1beta secretion. Cell. Physiol. Biochem. 43, 1113-1125. doi: 10.1159/000481753

Williams, N. C., and O'Neill, L. A. J. (2018). A role for the krebs cycle intermediate citrate in metabolic reprogramming in innate immunity and inflammation. Front. Immunol. 9:141. doi: 10.3389/fimmu.2018.00141

Wu, D., Han, R., Deng, S., Liu, T., Zhang, T., Xie, H., et al. (2018a). Protective effects of flagellin A N/C against radiation-induced NLR pyrin domain containing 3 inflammasome-dependent pyroptosis in intestinal cells. Int. J. Radiat. Oncol. Biol. Phys. 101, 107-117. doi: 10.1016/j.ijrobp.2018.01.035

Wu, D., Wu, K., Zhu, Q., Xiao, W., Shan, Q., Yan, Z., et al. (2018b). Formononetin administration ameliorates dextran sulfate sodium-induced acute colitis by inhibiting NLRP3 inflammasome signaling pathway. Mediat. Inflamm. 2018:3048532. doi: 10.1155/2018/3048532

Wu, P. J., Hung, Y. F., Liu, H. Y., and Hsueh, Y. P. (2017). Deletion of the inflammasome sensor Aim2 mitigates abeta deposition and microglial activation but increases inflammatory cytokine expression in an alzheimer disease mouse model. Neuroimmunomodulation 24, 29-39. doi: 10.1159/ 000477092

Xiao, H., Li, H., Wang, J. J., Zhang, J. S., Shen, J., An, X. B., et al. (2018). IL-18 cleavage triggers cardiac inflammation and fibrosis upon beta-adrenergic insult. Eur. Heart J. 39, 60-69. doi: 10.1093/eurheartj/ehx261

Yang, Z., Zhong, L., Xian, R., and Yuan, B. (2015). MicroRNA-223 regulates inflammation and brain injury via feedback to NLRP3 inflammasome after intracerebral hemorrhage. Mol. Immunol. 65, 267-276. doi: 10.1016/j.molimm. 2014.12.018.

Ye, X., Shen, T., Hu, J., Zhang, L., Zhang, Y., Bao, L., et al. (2017). Purinergic $2 \mathrm{X} 7$ receptor/NLRP3 pathway triggers neuronal apoptosis after ischemic stroke in the mouse. Exp. Neurol. 292, 46-55. doi: 10.1016/j.expneurol.2017. 03.002

Yuan, X., Wang, L., Bhat, O. M., Lohner, H., and Li, P. L. (2018). Differential effects of short chain fatty acids on endothelial Nlrp3 inflammasome activation and neointima formation: antioxidant action of butyrate. Redox. Biol. 16, 21-31. doi: 10.1016/j.redox.2018.02.007

Yue, N., Li, B., Yang, L., Han, Q. Q., Huang, H. J., Wang, Y. L., et al. (2018). Electroacupuncture alleviates chronic unpredictable stress-induced depressive- and anxiety-like behavior and hippocampal neuroinflammation in rat model of depression. Front. Mol. Neurosci. 11:149. doi: 10.3389/fnmol.2018.00149

Zhai, Y., Meng, X., Ye, T., Xie, W., Sun, G., and Sun, X. (2018). Inhibiting the NLRP3 inflammasome activation with MCC950 ameliorates diabetic encephalopathy in $\mathrm{db} / \mathrm{db}$ mice. Molecules 23:522. doi: 10.3390/molecules23030522

Zhan, X., Stamova, B., and Sharp, F. R. (2018). Lipopolysaccharide associates with amyloid plaques, neurons and oligodendrocytes in alzheimer's disease brain: a review. Front. Aging Neurosci. 10:42. doi: 10.3389/fnagi.2018.00042

Zhang, R.Y., Zhang, L., Zhang, L., Wang, Y.L., and Li, L. (2018). Anti-amyloidgenic and neurotrophic effects of tetrahydroxystilbene glucoside on a chronic mitochondrial dysfunction rat model induced by sodium azide. 72, 596-606. J. Nat. Med. doi: 10.1007/s11418-018-1177-y

Zhao, L. R., Xing, R. L., Wang, P. M., Zhang, N. S., Yin, S. J., Li, X. C., et al. (2018). NLRP1 and NLRP3 inflammasomes mediate LPS/ATPinduced pyroptosis in knee osteoarthritis. Mol. Med. Rep. 17, 5463-5469. doi: 10.3892/mmr.2018.8520

Zhou, K., Shi, L., Wang, Y., Chen, S., and Zhang, J. (2016). Recent advances of the NLRP3 inflammasome in central nervous system disorders. J. Immunol. Res. 2016:9238290. doi: 10.1155/2016/9238290

Zhou, K., Shi, L., Wang, Z., Zhou, J., Manaenko, A., Reis, C., et al. (2017). RIP1RIP3-DRP1 pathway regulates NLRP3 inflammasome activation following subarachnoid hemorrhage. Exp. Neurol. 295, 116-124. doi: 10.1016/j.expneurol. 2017.06.003

Zou, P., Liu, X., Li, G., and Wang, Y. (2018). Resveratrol pretreatment attenuates traumatic brain injury in rats by suppressing NLRP3 inflammasome activation via SIRT1. Mol. Med. Rep. 17, 3212-3217. doi: 10.3892/mmr.2017.8241

Conflict of Interest Statement: The authors declare that the research was conducted in the absence of any commercial or financial relationships that could be construed as a potential conflict of interest.

Copyright (C) 2018 Shao, Cao and Liu. This is an open-access article distributed under the terms of the Creative Commons Attribution License (CC BY). The use, distribution or reproduction in other forums is permitted, provided the original author(s) and the copyright owner(s) are credited and that the original publication in this journal is cited, in accordance with accepted academic practice. No use, distribution or reproduction is permitted which does not comply with these terms. 